\title{
Amylase production by solid state culture of Aspergillus oryzae on polyurethane foams. Some mechanistic approaches from an empirical model
}

\author{
M. A. Murado,* M. P. Gonzalez, A. Torrado and L. M. Pastrana \\ Instituto de Investigacions Marinas (CSIC), r/Eduardo Cabello, 6. Vigo-36208, Galicia, \\ Spain
}

*To whom correspondence should be addressed

\begin{abstract}
Using systems formed by particles of polyurethane foam of different densities soaked in an amylaceous effluent, the production of amylases by Aspergillus oryzae in solid state culture was studied by a complete factorial plan with four variables - support density, particle diameter, volume of liquid phase and substrate concentration. The empirical model obtained is consistent and allows a simple control of the response, superior in several respects to that of the corresponding submerged cultures. Moreover, it enables a mechanistic approach to the behaviour of the system, whose principal kinetic characteristics can be interpreted in terms of intra- and interparticular diffusional effects, which have a determining influence on basic state variables, such as water activity and gas transfer.
\end{abstract}

Keywords: empirical model, mechanistic interpretation, amylase production, solid state culture, polyurethane foam.

Introduction

Previous studies ${ }^{1-6}$ have shown that mussel processing wastes (MPW: average COD, 25 $\mathrm{g} \mathrm{O}_{2} /$ litre; main component glycogen, $10 \mathrm{~g} /$ litre), an important eutrophication factor in the Galician Rias Baixas estuarine system (NW of Spain), can be used as an adequate substrate for a number of microbial bioproductions of potential economic interest.

Solid state culture, the advantages and inconveniences of which have been indicated repeatedly, is now arousing a growing interest in fields such as delignification, ${ }^{7-9}$ the 
production of primary ${ }^{10-12}$ and secondary ${ }^{13-15}$ metabolites, the production of enzymes 16-19 and the modification of the limiting characteristics of submerged culture. ${ }^{20,21}$ In the particular case of MPW, the results obtained in the production of gibberellic acid in submerged culture ${ }^{3,4}$ were noticeably improved using systems formed by particles of corn cob soaked in the effluent. ${ }^{6}$ Moreover, the results obtained with this type of system permitted the definition of its response as a function of a series of physical factors, depending on the support, and manipulable in a simple and reproducible manner in order to efficiently modify the productive process.

Continuing with the study of processes in solid state with MPW as a culture medium, this paper emphasises the production of amylases by Aspergillus oryzae using particles of polyurethane foam of different densities as support. This study is intended to provide models allowing a mechanistic rather than empirical description of culture parameters.

Materials and Methods

General

Aspergillus oryzae (CBS 125-59), grown previously on MPW in submerged culture, was used as the test organism, ${ }^{2}$ The composition of the MPW and treatment to obtain the culture media employed has been described in detail in previous papers. ${ }^{2-4}$ In this case, the process involved: a) acidification of the effluent $(\approx 0.5 \mathrm{ml} 5 \mathrm{~N} \mathrm{HCI}$ per litre of effluent), b) clarification by separation of the protein precipitate spontaneously decanted after acidification, and c) ultrafiltration (cut off $100 \mathrm{kDa}$ ) of the clarified effluent to concentrations of glycogen between 10 and $100 \mathrm{~g} /$ litre.

The media thus obtained are characterised by their levels of polysaccharide and are named $\mathrm{nM}, \mathrm{n}$ being the concentration factor with regard to the clarified effluent or medium, M, with $10 \mathrm{~g} /$ litre of glycogen. The composition of essential nutrients of the most concentrated medium used $(10 \mathrm{M})$ was in g/litre: glycogen, 100; reducing sugars, 1.0-2.0; total nitrogen, $0-9-1.0$ (protein $\mathrm{N}, \approx 50 \%$ of total $\mathrm{N}$ ); total phosphorus, 0.08 0.10 . 
Before sterilisation (steam flow, $1 \mathrm{~h}$ ), the media were in all cases supplemented with $\mathrm{NaNO}_{3}$ and $\mathrm{KH}_{2} \mathrm{PO}_{4}$ to 1500 and $500 \mathrm{mg} /$ litre of $\mathrm{N}$ and $\mathrm{P}$, respectively, and the initial $\mathrm{pH}$ was adjusted to 6.0. Each experimental unit was inoculated with $20 \times 10^{6}$ spores suspended in an adequate volume of each culture medium, subsequently soaking the supports with the suspensions in sterile conditions. Incubations were carried out in 50 $\mathrm{ml}$ Erlenmeyer flasks in a water bath at $30^{\circ} \mathrm{C}$ for $24 \mathrm{~h}$.

Experimental design

In order to obtain an empirical model for the production of amylases in relation to the four variables that are specified in the following text, a complete first order orthogonal plan was used $\left(2^{4}\right)$, according to the procedures described by Akhnazarova \& Kafarov 22 and Box et al., ${ }^{23}$ with quadruple replication in the centre of the domain. Initially, the four variables and all their interactions (cross products) were considered potentially explanatory, leaving the possibility of enlarging the experimental plan in reserve to test, if necessary, the inclusion of quadratic terms in the model. The coefficients were calculated by standard multiple (orthogonal) regression, applying the ordinary least squares method (a preliminary test on the edges of the domain did not give evidence of heteroscedasticity) (see Table 3$)$. The Student $t$ test $(\alpha<0.05)$ was used as acceptance criteria for the coefficients, stepwise selection methods not being required due to the orthogonality of the independent variables matrix and, therefore, the absence of correlation between parameters (variance-covariance matrix diagonal).

The global consistency of the model was verified by Fisher's F test $(\alpha<0.05)$ applied to total error/experimental error and lack of fitting/experimental error quotients. The nonsignificant difference between the total average and the average in the centre of the domain, the high linear correlation between expected and observed values and the random distribution of residuals, dispensed with the need to consider quadratic terms. An additional confirmation of the proposed model, which is specifically discussed in the corresponding section, derives from its agreement with the results of subsequent trials, also presented in this paper.

The independent variables studied, the domains and coding criteria of which are given in Table 1, were: 
(1) Support density (p). The density of polyurethane foam depends on the proportions of reagents and the air injected into the mixture during the production process. Although in the context of this study it can be accepted that the density basically expresses the porosity (average size of pore) of the material, the former is better typified commercially than the second and provides more reproducible information. The domain of the variable was established in the $20-60 \mathrm{~g} / \mathrm{dm}^{3}$ interval.

(2) Particle diameter (Ø). Although cylindrical particles were used, with a diameter equal to the generatrix, it seemed acceptable to take this value as the diameter of an approximately equivalent sphere. Domain: 3-6 mm.

(3) Liquid phase in the system (L). This was defined as the initial volume of the spore suspension in the culture medium, measured in terms of $\mathrm{ml}$ per $\mathrm{g}$ of support. Domain: 6-12 ml/g.

(4) Substrate concentration (S). This was defined in terms of $\mathrm{mg}$ of glycogen per $\mathrm{ml}$ of liquid phase. Domain: 10-40 mg/ml.

Finally, the experimental units were charged with the same apparent volume (including interparticular space) of support, operationally defined by the measurement obtained when the material is charged in a test tube with a diameter $\approx 10$ times that of the particle, after compacting by gravity (tapping on the walls of the test tube in a manner identical to that when filling a chromatographic column). Following this procedure it was confirmed that, within the range of diameters and densities used, the relationships between weight and apparent volume were proportional to the densities indicated by the manufacturer.

Some peculiarities of this experimental plan are worth indicating. Firstly, it is reasonable to suppose that the carrying capacities of equal weights of polymers with different densities would be inversely correlated with their densities. The maximum loads of water retained by a unit of weight and volume of different foams were then determined by a series of compressions and expansions in a syringe following the procedure used for soaking a sponge. The results obtained (Table 2) confirmed the hypothesis. 
Secondly, it is clear that if the experimental units are equivalent in apparent volume of support, they differ in weight. Given the low density of the foams, the difference in weights at equal apparent volume seems a better solution than the opposite (variable apparent volume at constant weight) to obtain comparable units. One undesirable consequence of this solution perhaps is that it introduces the possibility of defining a variable ( $\mathrm{L} /$ maximum load or $\%$ of saturation of the liquid phase, $\lambda$ ) which does not coincide with that defined as L, possibly just as relevant and not orthogonal with respect to the other four. Nevertheless, it will be seen that such a non-orthogonal variable constitutes an additional way of verifying the relevance of those used.

Analytical methods

At pre-established times, the materials of each experimental unit were compressed in a syringe and washed repeatedly with distilled water. The extracts thus obtained were centrifuged at low speed and the analytical determinations were carried out in the corresponding supernatants, taken to suitable volumes. Total carbohydrates, protein, nitrogen, phosphorus, reducing sugars and total amylolytic activity were determined by methods described or referred to in previous papers. ${ }^{1,2}$ Non-volatile acids were determined by GLC after previous derivatisation with SYLON BTF (trimethylchlorosilane, SUPELCO), according to the method of Chapman \& Horvat. ${ }^{24}$

Results and Discussion

Evaluation of the empirical model

Table 3 summarises the results of the experimental plan in terms of the levels of total amylolytic activity per $\mathrm{ml}$ of liquid phase, after a $24 \mathrm{~h}$ incubation period (Y). Figure 1, which shows the time-course of a previous culture in conditions that are included in the domain used, reveals that this incubation time is suitable for establishing the necessary comparisons.

With the coefficients acceptable according to the Student $t$ test $(\alpha<0.05)$, the equation obtained is significant in Fisher's F tests $(\alpha<0.05)$ applied to the quotients total 
error/experimental error and lack of fitting/experimental error. This produces the following empirical model for the description of the system:

$\mathrm{Y}=19.93+1.82 \varnothing-3.98 \mathrm{p}+8.42 \mathrm{~S}+1.69 \varnothing \mathrm{L}-1.92 \varnothing \mathrm{pS}+1.48 \varnothing \mathrm{LS}(1)$

It is noteworthy that when increasing the requirements of the Student $t$ test to a level of $\alpha<0.02$, eqn (1) would be reduced to:

$\mathrm{Y}=19.93-3.98 \mathrm{p}+8.42 \mathrm{~S}-1.92 \varnothing \mathrm{PS}$

which also passes both Fisher tests at a level of $\alpha<0.05$, although it gives less adjusted predictions. Moreover, if the Student $t$ test is relaxed to the level of $\alpha<0.1$, it would be possible to accept several new interactions. Nevertheless, in no case does the L variable itself become significant, and the slight improvement obtained in the fitting of the predictions does not justify the additional complication. Therefore, the subsequent discussion is based on eqn (1). The corresponding standardised residuals are acceptable and lacking in suspicious regularities (Fig. 2), and the linear correlation between observed and expected values reflects the small second order component also detected through the difference between the average and central response, which is not significant regarding the second Fisher test.

The calculation carried out substituting variable $\mathrm{L}$ with $\lambda$ does not produce differences of interest in the results. Despite the lack of orthogonality, the equation obtained is significant with the same criteria applied to eqn (1). The difference in the definitions of $\mathrm{L}$ (dimensions $\mathrm{L}^{3} \mathrm{M}^{-1}$ ) and $\lambda$ (dimensionless) generates, in the non-orthogonal plan, additional terms involving the support density, but both equations are physically equivalent. Nevertheless, it should be noted that with the values used in the experimental plan, the substitution of $\mathrm{L}$ by $\lambda$ alters the domain, as shown in Fig. 3 . Finally, the suppression in the non-orthogonal case of the three terms with the lowest Student $\mathrm{t}$ (denoted $*$ in Table 4 ) leads, when calculating the equation once again, to the lack of significance of the other three interactions (denoted ** in Table 4). The calculation after the corresponding elimination reproduces the 'rough' model (eqn (2)). Thus, there do not seem to be any problems derived from possible ambiguities in the definition of the variables used.

Towards a mechanistic interpretation 
From consideration of eqn (1), the first noteworthy aspect is that, within the experimental domain, the effects of the variables selected can more than double the response in the centre (independent term), determining more than quadruple variation when considering the whole of the domain. The positive effect of the substrate, the consumption of which during the incubation period did not lead to limiting levels in any case, is the most important of the first order effects (Fig. 4(A)). Nevertheless, the decrease due to the association between high values of substrate and density should be noted (Fig. 4(B)). This is accentuated with high diameters, particularly with low liquid phases, and above all is a consequence of the negative term $Ø \mathrm{pS}$. The importance of $\varnothing \mathrm{pS}$ is also demonstrated by the fact that it is the only interaction which is maintained in the rough model (eqn (2)), and helps to explain the system in terms of the most characteristic structural factors of the solid state culture, i.e. water activity and oxygen transfer.

Bearing in mind the decisive role of oxygenation in the production of amylases (see Murado et al. $^{2}$ for data from submerged culture of the same species in the same medium as that used in this study), the principle factor in the behaviour described seems to derive from the characteristics of gaseous diffusion in the culture. Thus, the negative sign of all the terms involving density reflects the restrictions to intraparticular diffusion at high densities, which is logically accentuated by the increase in the size of the particle, as well as by high levels of substrate, which favour microbial growth and consequently the obstruction of the porous structure of the support.

Moreover, the participation of the diameter in three positive terms can be attributed to the favourable effect of the increase in particle size, at equivalent apparent volume, on interparticular diffusion. The fact that two of these terms are interactions which also include the liquid phase (the third is the first order effect of the diameter), indicates that high values of interparticular diffusion should be associated with high liquid phases, probably because this favours the maintenance of an adequate level of water activity, impeding its decrease to values that would excessively delay the metabolism. The liquid phase itself, although lacking a significant first order effect, forms part of the positive interactions. This suggests the requirement to maintain it, in subsequent experimentation, at least at the highest level of the domain tested. 
With regard to the effect of the concentration of the substrate on the production of the enzyme, it is interesting to note the growing linear tendency of the response in the relatively wide domain from 10 to $40 \mathrm{~g} /$ litre, There are several reasons that made this behaviour rather unlikely a priori:

(1) The usual asymptotic kinetics (Michaelis-Menten or Monod) implied in the generic problem of decreasing yields.

(2) The effects of the products of hydrolysis as inhibitors of the amylolytic activity (e.g. Aspergillus niger glucoamylase; Reddy \& Abouzied $^{25}$ ), or as repressors of enzymic synthesis (e.g. Endomyces fibuliger glucoamylase; Gonzalez et al. ${ }^{1}$ ). Both possibilities seem particularly interesting with respect to the present study, since according to Ramesh \& Lonsane, ${ }^{20}$ solid state culture reduces catabolic repression of $\alpha$-amylase in Bacillus licheniformis.

(3) The inhibition by substrate in solid state culture, similar to that described by Kumar \& Lonsane ${ }^{14}$ in relation to the production of gibberellic acid by Gibberella fujikuroi.

In spite of the potential interest in the second type of mechanisms, it does not appear reasonable to attribute an important role to them in the present case, as is supported by results previously obtained with the same strain of A. oryzae used in this study. ${ }^{1}$ Moreover, to determine the possible effect of the mode of culture on the relations between substrate and response, six new series of incubations were carried out, in which submerged cultures ( $250 \mathrm{ml}$ Erlenmeyer flasks with a $50 \mathrm{ml}$ load) were compared with solid state cultures $(\varnothing=1 ; p=-1 ; L=4$ in coded values $)$, at three levels of substrate in each case, and at equal initial concentrations of $\mathrm{N}$ and $\mathrm{P}$.

The results (Fig. 5) clearly showed the superiority of the solid state culture, both in production by volume unit of liquid phase and productivity relative to the consumption of the carbon source. Furthermore, at least at the highest concentration of carbohydrates, the consumptions of $\mathrm{N}$ and $\mathrm{P}$ were much faster in solid state than in submerged culture. Cessation in the consumption of carbohydrates and the change in the slope of the production of amylases detected in solid state at $40 \mathrm{~g} /$ litre, can reasonably be attributed to the deftciency of $\mathrm{N}$ and/or $\mathrm{P}$, the kinetics of which suggest levels become limiting at around $25 \mathrm{~h}$ of incubation. Therefore, the utilisation of larger supplements of $\mathrm{N}$ and $\mathrm{P}$ 
would have led (in solid state but not in submerged culture) to even higher amylase production than that observed.

On the other hand, while in submerged culture the levels of total amylolytic activity ceased to increase trom the intermediate concentration of the carbon source, in solid state this effect is not observed in spite of the aforementioned possible lack of $\mathrm{N}$ and $\mathrm{P}$. Such deficiencies complicate the comparison of both cases by the application of the Monod type model, since the condition of the sole limiting nutrient is not guaranteed from 24 (solid state) and $36 \mathrm{~h}$ (submerged). Nevertheess, the adjustment to such a model:

$\mathrm{TAA}=\frac{\text { TAAm } \times \text { TSo }}{\mathrm{Km}+\mathrm{TSo}}$

with TAA (total amylolytic activity) and TSo (total initial sugars) as variables, TAAm (maximum TAA) and $\mathrm{Km}$ (Monod constant) as parameters, and assuming that TAA $=0$ when TSo $=0$, is clearly acceptable (Fig. 6) as much before the deficiency as afterwards (production averages observed at 48 and $72 \mathrm{~h}$ ). Although, having examined only three concentrations for each operating mode, this description has only tentative value, it is not possible to ignore the remarkable difference (significant at $\alpha \leq 0.05$ ) observed in both parameters.

In accordance with this interpretation, the relationship between the initial concentration of the carbon source and amylase production is simply hyperbolic (decreasing yields) and does not show, in the time considered, effects of substrate inhibition. This valuation, suggested by Fig. 6 , was confirmed by adding a new term (Ki x $\mathrm{TS}^{2}{ }_{0}$, representative of the substrate inhibition) to the denominator of eqn (3). Again emphasising caution due to the small number of points available, it should be noted that the corresponding adjustment produces a non-significant $(\alpha \leq 0.05) \mathrm{Ki}$ coefficient with negative sign.

Finally, it is worthwhile to point out the different evolution of $\mathrm{pH}$ in both types of culture. Given the predominance of the $\alpha$-amylase in the amylolytic system of the strain tested and the sensitivity of this enzyme to acid pH (e.g. Gonzalez et al. ${ }^{1}$ ), this could 
suggest that the $\mathrm{pH}$ is the determining factor of the remaining differences observed between the submerged and solid state cultures. It would be necessary, therefore, to explain the origin of such differences in $\mathrm{pH}$.

In this respect, it could be hypothesised that the submerged culture induces a higher rate of accumulation of acid metabolites. However, this hypothesis could not be confirmed by GLC analysis of the corresponding media, using acetic, oxalic, pyruvic, gluconic and citric acids as standards. The chromatographic profiles, essentially identical in both types of culture, showed only small levels of some of these components, which were in no case over $100 \mathrm{mg} / \mathrm{litre}$.

On the other hand, if it is accepted that the initial decrease in $\mathrm{pH}$ is simply due to the use of carbohydrates in glycolysis, an alternative explanation is suggested for the different rates of consumption of the supplementary source of $\mathrm{N}\left(\mathrm{NaNO}_{3}\right)$. In fact, it is clear that in solid state the higher rate of consumption of $\mathrm{N}$ implies the reduction of $\mathrm{N}$ $\mathrm{NO}_{3}$ to $\mathrm{N}-\mathrm{NH}_{2}$ is faster, with the consequent consumption of protons. In the work of Ramesh \& Lonsane, ${ }^{20}$ however, an important role is attributed, in the specificity of the solid state culture, to a lower rate which, in conditions of low water activity, slows the diffusion of nutrients and metabolites, although this work refers to a bacterium (not a fungus) and to the aqueous diffusion of solutes. In contrast, the results relating to the production of amylases described in the present work suggest that, at least in this case, the specificity of the solid state seems to be related to more rapid metabolic rates, which, according to the empirical model obtained, depend on the factors which facilitate the oxygen transfer in these systems.

Acknowledgements

The authors would like to thank CICYT and Xunta de Galicia for their financial support (projects AMB 93-0136 and XUGA-40204B-92).

\section{REFERENCES}

1. Gonzalez Ma. P., Siso Ma. I. G., Murado, M. A., Pastrana, L., Montemayor Ma. I. \& Miron, J., Depuration and valuation of mussel-processing wastes. Characterisation 
of amylolytic postincubates from different species grown on an effluent. Bioresource Technol., 1992, 42(2), 133-140.

2. Murado, M. A., Siso Ma. I. G., Gonzalez Ma. P., Montemayor Ma. I., Pastrana, L. \& Pintado, J., Characterisation of microbial biomasses and amylolytic preparations obtained from mussel processing waste treatment. Bioresource Technol., 1993, 43(2), 117-125.

3. Murado, M. A., Gonzalez Ma. P., Pastrana, L., Siso Ma. I. G., Miron, J. \& Montemayor Ma. I., Enhancement of the bioproduction potential of an amylaceous effluent. Bioresource Technol., 1993, 44(2), 155-163.

4. Pastrana, L. M., Gonzalez Ma. P. \& Murado, M. A., Production of gibberellic acid from mussel processing wastes in submerged batch culture. Bioresource Technol., 1993, 45(5), 213-221.

5. Murado, M. A., Gonzalez, Ma. P. \& Pastrana, L. M., Mussel processing wastes as a fermentation substrate. In Fisheries Processing: Biotechnological Applications, ed. A. M. Martin. Chapman \& Hall, UK, 1993, pp. 311-343.

6. Pastrana, L. M., Gonzalez Ma. P., Pintado, J. \& Murado, M. A., Interactions affecting gibberellic acid production in solid state culture. A factorial study. Enzyme Microb. Technol., 1994, 17, 784-790.

7. Abdullah, A. L., Tengerdy, R. P. \& Murphy, V. G., Optimisation of solid substrate fermentation of wheat straw. Biotechnol. Bioeng., 1985, 27, 20-27.

8. Agosin, E., Jarpa, S., Rojas, E. \& Espejo, E., Solid state fermentation of pine sawdust by selected brown-rot fungi. Enzyme Microb. Technol., 1989, 11, 511-517.

9. Reid, I. D., Optimisation of solid-state fermentation for selective delignification of aspen wood with Phlebia tremellosa. Enzyme Microb. Technol., 1989, 11, 804-809.

10. Vassilev, N. B., Vassileva, M. Ch. \& Spassova, D. I., Production of gluconic acid by Aspergillus niger immobilized on polyurethane foam. Appl. Microbiol. Biotechnol., $1993,39,285-288$.

11. Soccol, C. R., Marin, B., Raimbault, M. \& Lebeault, J. M., Potential of solid state fermentation for production of L(+)-lactic acid by Rhizopus oryzae. Appl. Microbiol. Biotechnot, 1994, 41, 286-290.

12. Shankaranand, V. S. \& Lonsane, B. K., Coffee husk; an inexpensive substrate for production of citric acid by Aspergillus niger in a solid-state fermentation system. World J. Microbiol. Biotechnol., 1994, 10, 165-168. 
13. Kumar, P. K. R. \& Lonsane, B. K., Gibberellic acid by solid state fermentation: consistent and improved yields. Biotechnol. Bioeng., 1987, 30, 267-271.

14. Kumar, P. K. R. \& Lonsane, B. K., Potential of fed-batch culture in solid state fermentation for production of gibberellic acid. Biotechnol. Lett., 1987, 9, 179-182.

15. Kumar, P. R. K. \& Lonsane, B. K., Solid state fermentation: physical and nutritional factors influencing gibberellic acid production. Appl. Microbiol. Biotechnol., 1990, $34,145-148$.

16. Desgranges, C. \& Durand, A., Effect of $\mathrm{pCO}_{2}$ on growth conidiation and enzyme production in solid state culture of Aspergillus niger and Trichoderma viride TS. Enzyme Microb. Technol., 1990, 12, 546-551.

17. Rajaram, S. \& Varma, A., Production and characterisation of xylanase from Bacillus thermoalkalophilus grown on agricultural wastes. Appl. Microbiol. Biotechnol., 1990, 34, 141-144.

18. Ramesh, M. V.\& Lonsane, B. K., Critical importance of moisture content of the medium in $\alpha$-amylase production by Bacillus licheniformis M27 in a solid-state fermentation system. Appl. Microbiol. Biotechnol., 1990, 33, 501-505.

19. Soccol, C. R., Marin, B., Raimbault, M. \& Lebeault, J. M., Breeding and growth of Rhizopus in raw cassava by solid state fermentation. Appl. Microbiol. Biotechnol., 1994, 41, 330-336.

20. Ramesh, M. V. \& Lonsane, B. K., Ability of a solid state fermentation technique to significantly minimise catabolic repression of $\alpha$-amylase production by Bacillus licheniformis M27. Appl. Microbiol. Biotechnol., 1991, 35, 591-593.

21. Shankaranand, V. S. \& Lonsane, B. K., Ability of Aspergillus niger to tolerate metal ions and minerals in solid-state fermentation system for the production of citric acid. Process Biochem., 1994, 29, 29-37.

22. Akhnazarova, S. \& Kafarov, V., Experiment Optimisation in Chemistry and Chemical Engineering. MIR Publication, Moscow, 1982.

23. Box, G. E. P., Hunter, W. G. \& Hunter, J. S., Estadistica para Investigadores, ed. Reverte. Barcelona, 1989.

24. Chapman, G. W. \& Horvat, R. J., Determination of non-volatile acids and sugars from fruits and sweet potato extracts by capillary GLC and GLC/MS. J. Agric. Food. Chem., 1989, 37, 947-950. 
25. Reddy, C. A. \& Abouzied, M. M., Glucose feedback inhibition of amylase activity in Aspergillus sp. and release of this inhibition when cocultured with Saccharomyces cerevisae. Enzyme Microb. Technol., 1986, 8, 659-664. 
Table 1. Experimental domain and coding of the variables

\begin{tabular}{|c|c|c|c|c|c|c|c|}
\hline & \multirow[t]{2}{*}{$\begin{array}{l}\text { Coded } \\
\text { values }\end{array}$} & \multicolumn{2}{|c|}{$\begin{array}{l}\text { Orthogonal } \\
\text { variables }\end{array}$} & \multicolumn{2}{|c|}{ Natural values } & \multicolumn{2}{|c|}{$\begin{array}{l}\text { Non-orthogonal } \\
\text { variable } \lambda\end{array}$} \\
\hline & & $\begin{array}{l}\varnothing \\
(\mathrm{mm}\end{array}$ & $\mathrm{p}\left(\mathrm{g} / \mathrm{dm}^{3}\right)$ & $\begin{array}{l}\mathrm{S} \\
(\mathrm{mg} / \mathrm{ml})\end{array}$ & $\begin{array}{l}\mathrm{L} \\
(\mathrm{ml} / \mathrm{g})\end{array}$ & $\begin{array}{l}\text { coded } \\
\text { values }\end{array}$ & $\begin{array}{l}\text { natural } \\
\text { values }\end{array}$ \\
\hline & -1 & 3.0 & 20 & 10 & 6 & -1.00 & 13.2 \\
\hline & & & & & & -0.59 & 26.4 \\
\hline & 0 & 4.5 & 40 & 25 & 9 & -0.20 & 39.5 \\
\hline & & & & & & -0.19 & 39.6 \\
\hline & 1 & 6.0 & 60 & 40 & 12 & 1.00 & 78.9 \\
\hline Increments & 1 & 1.5 & 20 & 15 & 3 & & \\
\hline
\end{tabular}

Coding: $\mathrm{Vc}=(\mathrm{Vn}-\mathrm{Vo}) / \Delta \mathrm{Vn}$; decoding: $\mathrm{Vn}=\mathrm{Vo}+(\Delta \mathrm{Vn} \times \mathrm{Vc})$.

Vc: coded value; Vn: natural value; Vo: natural value in the centre of the domain; $\Delta \mathrm{Vn}$ : increment of $\mathrm{Vn}$ corresponding to one unit of $\mathrm{Vc}$.

Table 2. Maximum load of water (100\% saturation) in foams of different densities

\begin{tabular}{lll}
\hline & \multicolumn{2}{c}{ Water retained } \\
$\mathrm{p}\left(\mathrm{g} / \mathrm{dm}^{3}\right)$ & $\mathrm{ml} / \mathrm{g}$ & $\mathrm{ml} / \mathrm{dm}^{3}$ \\
\hline 20 & 45.42 & 908.4 \\
40 & 22.74 & 909.6 \\
60 & 15.19 & 911.4 \\
\hline
\end{tabular}


Table 3. Results of the experimental plan and analysis of the significance of the proposed model

\begin{tabular}{lllllllll}
\hline$\varnothing$ & $\mathrm{p}$ & $\mathrm{L}$ & $\mathrm{S}$ & $\mathrm{Y}$ & $\hat{\mathrm{Y}}$ & Coefficients & $\mathrm{t}$ & Model \\
\hline 1 & 1 & 1 & 1 & 22.50 & 27.43 & 19.93 & 53.287 & 19.93 \\
1 & 1 & 1 & -1 & 11.27 & 11.48 & 1.82 & 4.355 & 1.82 Ø \\
1 & 1 & -1 & 1 & 25.50 & 21.09 & -3.98 & 9.525 & $-3.98 \mathrm{p}$ \\
1 & 1 & -1 & -1 & 9.05 & 11.05 & 0.05 & 0.123 & NS L \\
1 & -1 & 1 & 1 & 44.00 & 39.24 & 8.42 & 20.146 & $8.42 \mathrm{~S}$ \\
1 & -1 & 1 & -1 & 15.69 & 15.60 & -0.56 & 1.334 & NS Øp \\
1 & -1 & -1 & 1 & 32.59 & 32.91 & 1.69 & 4.053 & 1.69 ØL \\
1 & -1 & -1 & -1 & 12.36 & 15.16 & 1.11 & 2.647 & NS ØS \\
-1 & 1 & 1 & 1 & 19.39 & 21.30 & -0.86 & 2.062 & NS pL \\
-1 & 1 & 1 & -1 & 6.86 & 3.56 & -0.68 & 1.635 & NS pS \\
-1 & 1 & -1 & 1 & 26.83 & 27.64 & -1.12 & 2.672 & NS LS \\
-1 & 1 & -1 & -1 & 5.13 & 4.00 & -1.08 & 2.579 & NS ØpL \\
-1 & -1 & 1 & 1 & 22.73 & 25.42 & -1.92 & 4.600 & -1.92 ØpS \\
-1 & -1 & 1 & -1 & 16.35 & 15.37 & 1.48 & 3.527 & 1.48 ØLS \\
-1 & -1 & -1 & 1 & 32.22 & 31.76 & -0.68 & 1.634 & NS pLS \\
-1 & -1 & -1 & -1 & 14.30 & 15.81 & -0.98 & 2.343 & NS ØpLS \\
0 & 0 & 0 & 0 & 21.95 & 19.93 & Average response $=19.93$ & \\
\hline & & & & & & & & \\
\hline
\end{tabular}




\begin{tabular}{|c|c|c|c|c|c|c|}
\hline 0 & 0 & 0 & 0 & 21.82 & 19.93 & Average central response $=20.44$ \\
\hline 0 & 0 & 0 & 0 & 19.09 & 19.93 & $\operatorname{Var}(\mathrm{Ee})=2.796$ \\
\hline \multirow[t]{7}{*}{0} & 0 & 0 & 0 & 18.90 & 19.93 & $\mathrm{t}(\alpha<0-05 ; v=3)=3.182$ \\
\hline & & & SS & $\mathrm{df}$ & QM & \\
\hline & & Model & 1581.66 & 6 & 263.61 & $\mathrm{QME} / \mathrm{QMEe}=3.166 \mathrm{~F}_{3}^{13}(\alpha=0.05)=8.740$ \\
\hline & & Error & 115.08 & 13 & 8.85 & $\mathrm{QMLF} / \mathrm{QMEe}=3.815 \quad \mathrm{~F}_{3}^{10}(\alpha=0.05)=8.790$ \\
\hline & & $\begin{array}{l}\text { Experimental } \\
\text { model }\end{array}$ & 8.39 & 3 & 2.80 & $r^{2}=0.932$ \\
\hline & & $\begin{array}{l}\text { Lack of } \\
\text { fitting }\end{array}$ & 106.69 & 10 & 10.67 & $\operatorname{adj} r^{2}=0.901$ \\
\hline & & Total & 1696.74 & 19 & & \\
\hline
\end{tabular}

Y: total amylolytic activity per ml of liquid phase; NS: non-significant coefficient; SS: sum of squares; QM: quadratic means; E: total error; Ee: experimental error; LF: lack of fit. 
Table 4. Alternative models, physically equivalent, derived from the two possible definitions of liquid phase in the system ${ }^{\mathrm{a}}$

\begin{tabular}{ll}
\hline Orthogonal (L) & Non-orthogon \\
\hline 19.93 & 19.93 \\
$1.82 \varnothing$ & $7.13 \varnothing$ \\
$-3.98 \mathrm{p}$ & $-3.99 \mathrm{p}$ \\
$8.42 \mathrm{~S}$ & $8.42 \mathrm{~S}$ \\
$1.69 \varnothing \mathrm{L}$ & $-6.28 \varnothing \mathrm{p} * *$ \\
$-1.92 \varnothing \mathrm{pS}$ & $7.41 \varnothing \lambda * *$ \\
$1.48 \varnothing \mathrm{LS}$ & $5.83 \varnothing \mathrm{S} * *$ \\
& $-6.38 \varnothing \mathrm{p} \lambda *$ \\
& $-6.97 \varnothing \mathrm{pS}$ \\
& $6.52 \varnothing \lambda \mathrm{S} *$ \\
& $-5.70 \varnothing \mathrm{p} \lambda \mathrm{S} *$
\end{tabular}

${ }^{\mathrm{a}}$ The suppression of the terms with lower Student $\mathrm{t}\left({ }^{*}\right)$ leads to a lack of significance $(* *)$ and a new calculation, which results in an equation equal to that in bold type.

Fig. 1. Time course of Aspergillus oryzae cultures on polyurethane foam (density $=60$ $\mathrm{g} / \mathrm{din}^{3}$; particle diameter $\left.=6 \mathrm{~mm}\right)$ soaked in MPW $(7.6 \mathrm{ml} / \mathrm{g})$. Initial total sugars: 10 (o) and $20(\bullet)$ g/litre. TS: total sugars, TAA: total amylolytic activity.

Fig. 2. Top: standardised residuals corresponding to the experimental matrix arranged according to increasing response values. Bottom: correlation between observed and expected values according to the model proposed.

Fig. 3. Variation of experimental domain in the plan of the variables $p$ and L (or $\lambda$ ), according to whether the criterion of definition of the liquid phase in the system determines an orthogonal (o) or non-orthogonal $(\bullet)$ plan (see text).

Fig. 4. Response surfaces corresponding to the TAA levels after $24 \mathrm{~h}$ as (A) a function of density and substrate, to extreme values of the diameter, with liquid phase high; (B) a function of diameter and liquid phase, to extreme values of density, with substrate high. Independent variables in coded values. 
Fig. 5. Comparative time course of A. oryzae submerged (open symbols) and solid state (dosed symbols) cultures at three initial concentrations of total sugars $(\Delta \mathbf{\Delta}: 10, \diamond \mathbf{\bullet} 20$ and $0 \bullet: 40 \mathrm{~g} /$ litre). TSc: total sugar consumption; Nt: total nitrogen; Pt: total phosphorus. Remaining notes as in Fig. 1.

Fig. 6. Total amylolytic activity levels as a function of initial concentrations of total sugars in submerged (o) and solid state $(\bullet)$ cultures. Left: before the possible deficit of $\mathrm{N}$ and $\mathrm{P}$ ( $24 \mathrm{~h}$ in solid state and $36 \mathrm{~h}$ in submerged culture). Right: after the possible deficit (average values of the levels at 48 and $72 \mathrm{~h}$ ). 